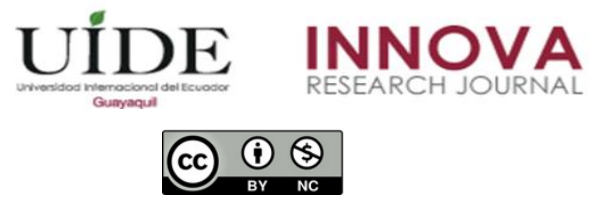

INNOVA Research Journal, ISSN 2477-9024

(Septiembre-Diciembre 2019). Vol. 4, No.3.1 pp. 108-125

DOI: https://doi.org/10.33890/innova.v4.n3.1.2019.1223

URL: http://revistas.uide.edu.ec/index.php/innova/index

Correo: innova@uide.edu.ec

\title{
Factores de apoyo de la Academia para generar emprendimientos, caso de estudio Facultad de Hospitalidad y Servicios de la Universidad UTE
}

\section{Factors of support of the Academy to generate Entrepreneurships, case study School of Hospitality and Services of the UTE University}

Mauricio Balarezo Noboa

Universidad Tecnológica Equinoccial, Ecuador

Héctor Cumbal Flores

Universidad de las Fuerzas Armadas ESPE, Ecuador

Autor para correspondencia: mauricio.balarezo@ute.edu.ec; hscumbal@espe.edu.ec

Fecha de recepción: 11 de julio del 2019 - Fecha de aceptación: 30 de septiembre del 2019

\section{Resumen}

El 27.6\% de desempleados pertenecen a jóvenes con estudios superiores. El emprendimiento es una oportunidad de trabajo para quienes sueñan en tener una empresa, pero no es fácil, considerando que las IES no cuentan con las estrategias para convertirse en un aliado eficiente. Como objetivos se plantearon: conocer la actitud emprendedora de los estudiantes y descubrir aquellos factores de apoyo provenientes de la academia para que un proyecto estudiantil se convierta en empresa. Se utilizó el método exploratorio descriptivo, estudio de caso en la Facultad de Hospitalidad y Servicios, de la Universidad UTE, como técnicas: encuestas a 316 estudiantes; el cuestionario se basó en el Entrepreneurial Attitude Orientation (EAO) Scale. Como resultados, el $63 \%$ de los estudiantes indicaron que están dispuestos a desarrollar nuevas oportunidades, el $43 \%$ señalaron que el mayor impedimento para emprender es la capacidad económica, el apoyo más importante que esperan de la universidad es capital de preincubación 38\%. Los resultados obtenidos suponen que la facultad debe implementar estrategias para el desarrollo de una cultura emprendedora como base para la generación de proyectos innovadores. Estudiantes, universidad, empresa y gobierno deben interactuar en el marco de un ecosistema de negocios y redes con alcance nacional y regional.

Palabras Claves: negocio; empresa; desarrollo económico y social; gestión

\begin{abstract}
$27.6 \%$ of unemployed belong to young people with higher education. Entrepreneurship is a job opportunity for those who dream of having a company, but it is not easy, considering that IES do not have the strategies to become an efficient ally. The objectives were: to know the entrepreneurial attitude of the students and discover those support factors from the academy so that a student project becomes a company. The descriptive exploratory method, case study in the Faculty of Hospitality and Services, of the UTE University, was used as techniques: surveys of 316 students; the questionnaire was based on the Entrepreneurial Attitude Orientation (EAO) Scale. As a result, $63 \%$ of the students indicated that they are willing to develop new opportunities, $43 \%$ indicated
\end{abstract}


that the greatest pitfall to entrepreneurship is economic capacity, the most important support they expect from the university is $38 \%$ preincubation capital. The results obtained mean that the faculty must implement strategies for the development of an entrepreneurial culture as the basis for the generation of innovative projects. Students, university, business and government must interact within the framework of a business and network ecosystem with national and regional reach.

Key words: business; company; social and economic development and organizations

\section{Introducción}

Según el INEC, en el año 2018 sumados el subempleo y desempleo en Ecuador son 1.619.821 personas, según datos de SENESCYT el 27.6\% de desempleados pertenecen a jóvenes con estudios superiores, es decir que aproximadamente 447.071 personas desempleadas son jóvenes egresados o graduados de Instituciones de Eduación Superior IES.

La universidad es un actor clave en el desarrollo del perfil profesional de sus egresados, es el contexto idóneo para mejorar la empleabilidad de los mismos a través del fomento al emprendimiento, que favorezca la elevada tasa de desempleo juvenil en Ecuador, que casi supera el 60\%. (Jacóme, Tinajero, \& Suárez, 2018). Los egresados de la educación superior están dentro de estas estadísticas y preocupa el hecho que, en determinadas carreras, egresen y no encuentren trabajo, es inquietante además haber estudiado y ocuparse en actividades distintas a las de su formación profesional.

El emprendedor empresario es una persona que identifica una oportunidad de negocio y organiza los recursos necesarios para ponerlo en marcha (Hidalgo, 2015). Entrepreneurship es un término anglosajón de uso generalizado que hace referencia al emprendedor y a la creación de empresas. El empresario emprendedor es alguien que ya nace así, que tiene visión para los negocios y para las oportunidades, que compite agresivamente, que actúa con firmeza sobre las cosas tangibles y hacia la acción. El concepto de espíritu emprendedor es amplio y no necesariamente se centra en la tarea de crear una empresa, sino que implica también estar en un proceso de buscar y descubrir nuevas oportunidades de negocio. El ser emprendedor implica una manera de pensar, es un estado de la mente, un conjunto de comportamientos caracterizados por innovación, flexibilidad y creatividad. Cada acción de emprender es el final de un proceso y el inicio de otro, aunque la acción de emprender más obvia es la creación de una nueva empresa (Espíritu Olmos \& Sastre Castillo, 2007).

El Consejo Europeo de Lisboa de marzo de 2000 definió la educación en el espíritu emprendedor como el motor necesario para una cultura empresarial más dinámica, y el Consejo de Ministros de Educación de la UE, de febrero de 2001, señaló entre los objetivos futuros de los sistemas educativos el desarrollo del espíritu de empresa en la educación (Espíritu Olmos \& Sastre Castillo, 2007).

Estos cuatro aspectos fundamentales; el divorcio de las universidades con el sector privado, el exceso de tramitología, la ausencia de capital de riesgo y un sistema de protección intelectual que todavía no está acostumbrado a un ritmo dinámico de innovación en el país, evidencian que el sistema de incubación de empresas del Ecuador está luchando con procesos tradicionales poco adaptables a los cambios (Emprendedora, 2006). 
En este contexto, es oportuno conocer a dos de los actores importantes en el tema del emprendimiento universitario, por un lado, los estudiantes con sus ideas y sus expectativas y por otro, la universidad con su importante apoyo y sus auténticas capacidades para convertir estas ideas en empresas exitosas.

El presente estudio es de carácter cualitativo, exploratorio - descriptivo y tiene como como objetivos conocer la actitud emprendedora de los estudiantes y descubrir aquellos factores de apoyo provenientes de la academia para que un proyecto de negocio estudiantil se convierta en una empresa exitosa.

Luego de esta introducción, el trabajo indica la problemática, luego el marco teórico de referencia; enseguida se detalla el método de investigación; posteriormente se presentan los resultados y la discusión del estudio de caso, finalmente se esbozan las conclusiones y las implicaciones futuras que trae esta investigación.

\section{Problemática}

En la universidad, la rutina transcurre entre preparar clases y tareas, en este valioso tiempo es difícil percatarse que los estudiantes tienen ideas de negocio con mucho potencial.

En este sentido, la universidad no cuenta con mecanismos apropiados para enfocar estas iniciativas a un sistema que oferte diversas estrategias de apoyo, de modo que el estudiante cuente con un ambiente propicio y recursos suficientes que fomenten la creación de una empresa y que se sostenga en el tiempo.

Otro problema es que los estudiantes que no tienen ninguna actitud hacia el emprendimiento, legítimamente, buscan una oportunidad en el mercado laboral, por las condiciones económicas críticas simplemente no encuentran trabajo y se ven obligados a ocuparse en actividades impensadas, causando así frustración en los egresados que por necesidad tienen que dedicarse a tareas distintas a las de su formación profesional, integrándose así a filas del desempleo o subempleo.

\section{Estrategias de apoyo en Universidades para el emprendimiento}

El proceso de emprendimiento juvenil puede ser entendido desde varias aristas complejas. En México, un estudio aborda esta complejidad clasificando en fenómenos: administrativo, sociológico, psicológico y político. La Tabla 1 muestra los temas de emprendimiento empresarial juvenil por cada fenómeno: 


\section{Tabla 1}

Temas relacionados al emprendimiento empresarial por disciplina.

\begin{tabular}{|c|c|c|c|}
\hline $\begin{array}{c}\text { Fenómeno } \\
\text { administrativo }\end{array}$ & $\begin{array}{l}\text { Fenómeno } \\
\text { sociológico }\end{array}$ & $\begin{array}{l}\text { Fenómeno } \\
\text { psicológico }\end{array}$ & Fenómeno político \\
\hline $\begin{array}{l}\text { Proceso de conducción de } \\
\text { la organización (PODC) } \\
\text { (PHVA) }\end{array}$ & $\begin{array}{c}\text { Empresa como } \\
\text { organización social }\end{array}$ & Autoeficacia & $\begin{array}{l}\text { Políticas públicas de apoyo } \\
\text { al emprendedurismo }\end{array}$ \\
\hline Plan de negocios & Valores & Capacidades & Generación de empleos \\
\hline Marketing & $\begin{array}{l}\text { Recursos } \\
\text { humanos }\end{array}$ & Habilidades & Aportación al PIB nacional \\
\hline $\begin{array}{l}\text { Responsabilidad social } \\
\text { empresarial }\end{array}$ & $\begin{array}{l}\text { Relaciones } \\
\text { sociales }\end{array}$ & Competencias & $\begin{array}{l}\text { Presupuestos etiquetados } \\
\text { para apoyo al } \\
\text { emprendedurismo }\end{array}$ \\
\hline Recursos humanos & $\begin{array}{l}\text { Redes de apoyo } \\
\text { (moral) }\end{array}$ & $\begin{array}{l}\text { Intenciones de } \\
\text { emprendimiento }\end{array}$ & $\begin{array}{l}\text { Financiamiento } \\
\text { gubernamental }\end{array}$ \\
\hline $\begin{array}{l}\text { Financiamiento por parte } \\
\text { de la banca privada }\end{array}$ & $\begin{array}{c}\text { Educación en } \\
\text { emprendimiento }\end{array}$ & $\begin{array}{l}\text { Experiencia y } \\
\text { formación }\end{array}$ & $\begin{array}{l}\text { Facilidades y flexibilización } \\
\text { de aspectos legales }\end{array}$ \\
\hline Uso de TIC's & Prestigio & Liderazgo & $\begin{array}{l}\text { Institucionalidad } \\
\text { emprendedora }\end{array}$ \\
\hline Aspectos contables & Estatus & $\begin{array}{l}\text { Inteligencia } \\
\text { emocional }\end{array}$ & Habilidades directivas \\
\hline Desarrollo e investigación & $\begin{array}{l}\text { Modelos de rol } \\
\text { emprendedor }\end{array}$ & $\begin{array}{l}\text { Personalidad } \\
\text { proactiva }\end{array}$ & Relaciones de poder \\
\hline
\end{tabular}

Fuente: Víctor García García

En México el emprendimiento empresarial juvenil es muy importante de reconocer por cuanto contribuye para reducir las cifras de desempleo, sin embargo no existe una cultura empresarial juvenil debido a las siguientes causas: falta de oportunidades para los jóvenes $42 \%$, no existe una calidad educativa que la fomente $29 \%$, en otro sentido, los jóvenes están dispuestos a superarse $25 \%$, a esto se suma el desconocimiento marcado por parte de los jóvenes respecto a las políticas públicas que incentivan el emprendimiento (García-García, 2015).

En el caso del Tecnológico de Monterrey es destacable que hablan de una Universidad Socialmente Responsable, en donde hay todo por hacer en cuanto al impulso de los emprendimientos estudiantiles pues existe gran número de alumnos que formar y un buen número de instituciones que desean apoyar. Hay que trabajar en red, con mecanismos como centros de transferencia de conocimientos en donde se articulan los esfuerzos de cada campus, identificando y atendiendo con diferentes estrategias necesidades específicas. El programa de incubadoras de empresas del Tec de Monterrey se ha posicionado gracias a la creación de tres redes de incubadoras, clasificadas en: Red de Incubadoras Sociales, Red de Tecnología Intermedia y Red de Tecnología Avanzada. Este programa fue diseñado para apoyar a los alumnos, egresados y comunidad en general para apoyar las ideas de negocio y convertirlas en empresas exitosas a nivel nacional e internacional. El gobierno mexicano también contribuye al ordenamiento de los emprendimientos, a través de la Secretaría Federal de Economía, esta institución señaló en el año 2006 que el 80\% de las micro, pequeñas y medianas empresas que se crearon sin consultoría desaparecieron antes de los dos años, considerando que este grupo representa el 99,8\% de la estructura económica mexicana. Con el reconocimiento que este grupo de empresas es un aporte importante se crea el llamado Sistema Nacional de Incubadoras de Empresas, con la finalidad de constituir empresas de alto potencial a nivel nacional e 
internacional. En el Tecnológico de Monterrey hay casos de éxito en empresas que se han desarrollado con la iniciativa estudiantil mientras estudiaban su carrera o cuando egresaron, estas iniciativas tienen que ver con proyectos de tecnología intermedia y avanzada. (Saldaña \& Coutiño, 2010).

Quien emprende aprovecha las oportunidades que se le puede presentar, las mismas que son asumidas como una opción superior de autorrealización y de generación de empleo e ingresos (Perez, 2009). En el Perú dos de cada tres empresas que se crean cierran antes de un año de funcionamiento, considerando que la autoridad de control registra 300.000 empresas por año. Los negocios de los jóvenes fracasan porque no están preparados para iniciar un negocio.

En Perú, aunque existe una cantidad importante de universidades, pocas forman líderes emprendedores, más bien se preocupan en formar profesionales para que se empleen bajo dependencia de empresas ya constituidas. Las universidades que sí incentivan el espíritu emprendedor lo hacen a través de incubadoras.

El marco legal y la disposición de recursos a favor del emprendimiento son estrategias de apoyo para que las ideas de negocio se conviertan en empresa. Se fomenta con estas políticas que las universidades manejen entidades empresariales de su propiedad.

Las universidades han encontrado mecanismos para la creación de nuevas empresas como por ejemplo las empresas derivadas, denominadas spin: start up (creación a partir de la invención y licencias), spin off como inversión de una universidad en el patrimonio de una empresa creada a partir de una innovación (Orozco \& Chavarro, 2008).

La universidad que apoya el emprendimiento despliega varias estrategias para el trabajo con el gobierno y la industria en el desarrollo de actividades innovadoras, se involucran en redes con instituciones públicas y también privadas en un contex to de mercado nacional e internacional. En España universidades incluyeron una nueva misión: promoción de la cultura emprendedora dirigida a toda la comunidad universitaria (académicos, estudiantes) iniciando con modificaciones en la normativa de educación superior (Guerrero Maribel \& Urbano David, 1992).

En Colombia, uno de los paradigmas que comparte la academia se refiere al desarrollo de la capacidad empresarial y la creación de empresas, aquí el emprendimiento se vincula con algunos factores como riqueza, empresa, empleo, valor, crecimiento, pero posiblemente, la perspectiva más asociada es la innovación. Para el fomento del emprendimiento, en este país, existe una normativa que indican varios factores de apoyo en los aspectos: educativos, culturales, económicos, políticos y legales. En cuanto a los factores incluidos en el aspecto educativo de esta normativa destacan: 1) promoción del espíritu emprendedor en el sistema educativo; 2) integración del sistema educativo y el sistema productivo; 3 ) formación de competencias para el emprendimiento en los programas académicos; 4) evaluación de la calidad de los programas de fomento al emprendimiento en el sistema educativo; 5) generación de un esquema de formación de formadores en el tema de emprendimiento; 6) creación de procesos de capacitación empresarial; 7) incorporación del emprendimiento a los currículos (preescolar a educación media); 8) generación de eventos de emprendimiento en el sistema educativo; 9) creación de la 
opción de grado universitario mediante un plan de negocio (Sanabria Rangel, Morales Rubiano, \& Ortiz Riaga, 2015).

La estrategia para el proceso de innovación se fundamenta en 6 pilares: innovación y emprendimiento, entorno de la competitividad, ciencia y tecnología, gestión de negocios, recursos para el financiamiento y el entorno institucional, siendo este último la base sobre la cual se asientan el resto de pilares y es donde se encuentra la universidad. La estrategia de ciencia, tecnología e innovación en Colombia ha estado alejada del sector empresarial, la Tabla 2 señala los retos de este país para consolidar la estrategia en base a modelos educativos por tipo de sector.

\section{Tabla 2}

Modelos educativos por sector

\begin{tabular}{|c|c|c|}
\hline $\begin{array}{l}\text { Entorno } \\
\text { institucional }\end{array}$ & $\begin{array}{l}\text { Más compromiso del gobierno y las autoridades } \\
\text { locales con la CT\&I }\end{array}$ & $\begin{array}{l}\text { Reorientar los roles de las } \\
\text { entidades públicas que hacen } \\
\text { parte del Sistema de CT\&I }\end{array}$ \\
\hline Financiación & $\begin{array}{l}\text { Incrementar los recursos públicos, especialmente } \\
\text { para financiar proyectos de innovación empresarial }\end{array}$ & $\begin{array}{l}\text { Mejorar el proceso de } \\
\text { asignación y distribución de } \\
\text { recursos para CT\&I del Sistema } \\
\text { General de Regalías }\end{array}$ \\
\hline $\begin{array}{l}\text { Cultura de } \\
\text { innovación }\end{array}$ & $\begin{array}{l}\text { Convertir la empresa en el centro de la estrategia de } \\
\text { CT\&I }\end{array}$ & $\begin{array}{l}\text { Promover el surgimiento de } \\
\text { emprendimientos 'spin off' de } \\
\text { universidades y centros de } \\
\text { investigación }\end{array}$ \\
\hline $\begin{array}{l}\text { Ciencia y } \\
\text { tecnología }\end{array}$ & $\begin{array}{l}\text { Financiar, apoyar y conectar a grupos de } \\
\text { investigadores }\end{array}$ & $\begin{array}{l}\text { Promover centros de } \\
\text { investigación sectoriales y } \\
\text { transversales de talla mundial }\end{array}$ \\
\hline Capital humano & $\begin{array}{l}\text { Aumentar la participación de las ciencias básicas, } \\
\text { diseño e ingenierías en la educación superior }\end{array}$ & $\begin{array}{l}\text { Fomentar la incorporación de } \\
\text { investigadores en empresas y } \\
\text { técnicos y tecnólogos en pymes }\end{array}$ \\
\hline
\end{tabular}

Fuente: Cuadernos Fedesarrollo

En Colombia se reconoce la alianza entre, industria, gobierno y universidad, con el interés común de generar desarrollo económico competitivo teniendo a la innovación como protagonista de estos esfuerzos. Como estrategia esencial se encuentra la promoción de la asociatividad y el fortalecimiento de las pymes para el desarrollo de proyectos de ciencia, tecnología e innovación de impacto regional. Se priorizan recursos públicos para financiar iniciativas empresariales que involucren la cooperación entre la empresa, universidad y centros de investigación, el estímulo debe ser direccionado también a los proveedores dentro de una cadena productiva. Otra estrategia clave es destinar recursos a través de convocatorias para impulsar empresas spin off de innovación. La capacitación en temas de fortalecimiento empresarial, asuntos legales, de propiedad intelectual, desarrollo de prototipos se podría impartir considerando posibles convenios con determinadas facultades universitarias según la especialidad y necesidad (Hernando \& Mitchell, 2017).

\section{La cultura del emprendimiento}

La cultura emprendedora se entiende como un proceso que se compone de los siguientes elementos: creatividad, innovación, responsabilidad y emprendimiento, esta cultura se 
fundamenta en habilidades, conocimiento y actitudes, es decir, quien emprende debe tener competencia para ello. Cuando la creatividad investigadora, el desarrollo innovador y la transferencia emprendedora se integran, entonces se construye la cadena de valor Investigación científica + Desarrollo tecnológico + Transferencia emprendedora, I+D+i (De la Fuente Arias, Vera Martínez, \& Cardelle-Elawar, 2017).

Algunos estudios señalan una serie de características para lograr el emprendimiento y la innovación, agrupadas en las siguientes dimensiones: formación académica, infraestructura, personalidad, actividades y estrategias. Las características dentro de la dimensión de formación académica sobresalen: desarrollo de plan de negocios, creación de empresas y pequeños negocios, habilidades de dirección y competencia, enfoque en promover la autonomía, comprensión y estudio de la problemática Pymes, inclusión en los currículos sobre protección de derechos de autor, propiedad intelectual y explotación comercial de aquella propiedad intelectual, comunicación efectiva. En lo que respecta a la personalidad característica del emprendedor se tiene: necesidad de independencia, toma de riesgos, el deseo de resolver problemas y lograr satisfacción con el logro de objetivos, audacia controlada, construye el camino (Montoya \& Casas, 2015).

Una situación a considerar es el incentivo que debe destinarse a los estudiantes para que su creatividad sea aceptada. Las razones por las cuales inician los emprendimientos se relacionan con su motivación por desarrollar proyectos con base en la innovación, ya que los emprendedores están convencidos de que ello les permitirá crecer y consolidarse (Liévano, Vargas, Pico, \& Pérez, 2018).

\section{La actitud emprendedora}

La actitud emprendedora de los universitarios se puede identificar por los rasgos psicológicos y de personalidad. Algunas instituciones de educación superior han montado programas de capacitación en formación empresarial, quienes reciben estos programas tienen mayor actitud hacia el espíritu emprendedor, por lo que las universidades son el recinto importante para implementar estas estrategias a favor de los emprendimientos universitarios.

Los estudiantes no miran con optimismo el emprender pues se presentan varias complicaciones como falta de capacidad financiera para enfrentar los gastos operacionales, personal, impuestos y otras erogaciones que no son posibles enfrentarlas en momentos de inicio y en un ambiente marcado por la incertidumbre, en este sentido el estudiante prefiere emplearse en empresas existentes por temas de seguridad y de menos responsabilidad. El universitario considera vital que las universidades implementen un modelo de enseñanza enfocado al emprendimiento para asimilar una teoría consolidada y una visión empresarial clara (EcheverriSanchez, Valencia-Arias, Benjumea-Arias, \& Barrera-Del Toro, 2018).

Diversas estrategias de gobierno, con sus políticas, pretenden formar más empresarios coadyuvando a que nuevas generaciones de profesionales tengan mayor actitud al emprendimiento, pero en actividades competitivas. La oferta académica es una contribución importante afín al desarrollo del emprendimiento y es una alternativa palpable de las universidades para el progreso de la región (Tarapuez, García, \& Castellano, 2018). 
La mayor parte de estudiantes se caracteriza por una actitud favorable a emprender, presentándose una relación apreciable con variables de edad, de estudios, unidad académica y con el sector que eligen (Canto \& Herrera, 2018).

La Tabla 3 señala la caracterización de los universitarios en Quindío, Colombia, agrupándolos en cuatro dimensiones:

\section{Tabla 3}

Caracterización de los estudiantes universitarios del Quindío

\section{Emprendedores y futuros emprendedores}

- Tienen o tuvieron una empresa o muestran una seria intención emprendedora.

- Los estudios de sus madres van desde no haber ido al colegio hasta posgrado.

- Sus progenitores son empresarios.

- Están casados y su edad es superior a 28 años.

- Estudian carreras del área de Ciencias

Económicas y Administrativas.

- Tienen familiares empresarios y experiencia laboral.

- Pertenecen a la clase alta.

\section{Relevo generacional en la empresa}

- Tienen planes de vincularse a una empresa de propiedad de su familia.

- Sus progenitores han alcanzado título profesional o de postgrado.

- Están vinculados con las universidades: Gran

Colombia, Von Humboldt y San Buenaventura.

- Estudian carreras de las áreas de Ciencias de la

Salud, Ingeniería y Arquitectura.

- Son los más jóvenes, su edad es hasta 23 años.

\section{Indeterminados}

- No asumen posición sobre la intención emprendedora.

- Sus madres no fueron al colegio o estudiaron primaria y se dedican al hogar o a labores independientes.

- Sus padres estudiaron primaria o secundaria.

- Tienen entre 24 y 27 años de edad.

- Están vinculados a la Universidad del Quindío.

- Estudian carreras de pedagogía.

Fuente: Innovar

Según estas características los universitarios con actitud emprendedora muestran una seria intención de emprender, tienen responsabilidad en un hogar conformado, estudian en una carrera relacionada con la rama económica y administrativa, esta caracterización se relaciona además con el relevo generacional en la empresa. Quienes tienen características en las dimensiones indeterminados y empleómanos muestran poca intención a emprender. En el contexto universitario brasileño un estudio reveló que la actitud personal (entendida como el grado en que un individuo tiene una evaluación positiva o no en ser emprendedor) la investigación presentó un 13\% que significa que hay una evaluación positiva por parte de los alumnos en el sentido de que se vuelvan emprendedores, pero esto implica además que el ambiente para el emprendimiento necesita fortalecerse por parte de las universidades y con políticas públicas provenientes del propio gobierno y así atraer a la juventud universitaria hacia el emprendimiento (Souza \& Silveira, 2018).

En la Universidad Central del Ecuador también se desarrolló un estudio sobre la actitud 
emprendedora, el instrumento fue desarrollado en base al modelo de medición de Likert, en donde se detallan 6 dimensiones claves de la actitud emprendedora: proactividad, ética profesional, empatía, innovación, autonomía y toma de riesgos. Se procedió a identificar la actitud emprendedora de los estudiantes y en función a la evaluación Alpha de Crombach's se estableció como resultado que cada uno de los estudiantes puede llegar a tener en el transcurso de su vida universitaria y posteriormente a su vida profesional una actividad emprendedora en acción. Se debe fortalecer la investigación en el emprendimiento e innovación que motive en forma efectiva la actitud emprendedora en los jóvenes de la universidad y también se requiere capacitar a los catedráticos universitarios en temas relacionados a la cultura del emprendimiento (Revelo, Lucio, Mata, \& Romero, 2017).

Aunque las mallas curriculares fomentan el espíritu emprendedor, los estudiantes ecuatorianos, no se encuentran totalmente seguros de emprender. La única dimensión que incide en forma significativa, sobre el atributo de emprendimiento es la variable Familiares, en consecuencia, el hecho que el estudiante provenga de una familia de empresarios es, precisamente el inductor de la conducta emprendedora de los estudiantes del último año de una universidad del Estado en la provincia del Guayas (Attitude, Students, The, \& Year, 2018) .

\section{Metodología}

Esta investigación es de carácter cualitativo, exploratorio - descriptivo. Para evaluar la actitud emprendedora se aplicó un cuestionario a los estudiantes del tercer al noveno nivel de las carreras de Gastronomía, Hotelería y Turismo de la facultad de Hospitalidad y Servicios de la Universidad UTE, a una muestra de 316 estudiantes de una población de 465, determinada con un nivel de confianza del $99 \%$ y un margen de error del $5 \%$.

El cuestionario se basó en el Entrepreneurial Attitude Orientation (EAO) Scale, desarrollado en Estados Unidos por Robinson, quien para este fin determina cuatro factores en el ámbito de los negocios: Necesidad de logro, Innovación, Control Interno y Autoestima, (Robinson, Stimpson, Huefner, \& Hunt, 2018). Estos factores son explicados, además, en el trabajo de titulación "Actitudes emprendedoras de los estudiantes universitarios: Caso Universidad Católica de Santiago de Guayaquil”, (Marquinez, 2018), así:

Factor necesidad de logro en los negocios, referido a resultados concretos con la creación y desarrollo de nuevos emprendimientos, mide la predisposición de los estudiantes al trabajo independiente y esfuerzo extraordinario junto con la capacidad de manejar un negocio propio con una sólida actitud ante la incertidumbre. Para evaluar este factor se propusieron las siguientes opciones en la encuesta:

- Creo que la gente con éxito se manejará mejor que yo en reuniones de negocios.

- Las horas regulares de trabajo y las vacaciones son más importantes para mí que las nuevas oportunidades y retos que ofrezca un puesto.

- Creo que para tener éxito en un negocio debes dedicar tiempo todos los días a desarrollar nuevas oportunidades.

- Creo que es necesario pasar mucho tiempo planeando las actividades empresariales.

- Me siento bien cuando he trabajado duro para mejorar mi trabajo 
Factor de innovación en los negocios, percibido como el funcionamiento de una empresa en una forma nueva y única, así como saber asumir riesgos, aceptar situaciones de incertidumbre. Para la evaluación se plantearon los siguientes enunciados:

- Me gusta que las cosas sean estables y predecibles.

- Me gusta trabajar donde no hay mucha incertidumbre ni cambios constantes.

- No me molesta la incertidumbre y el riesgo que suele provocar lo desconocido.

- Suelo apostar por buenas ideas, aunque no sean del todo seguras.

- Tengo mucha necesidad de nuevas aventuras.

- Lo que verdaderamente me motiva es pensar en nuevas ideas que estimulen un negocio.

- Por lo general evito asumir riesgos.

- Vivo cómodo en situaciones de cambio e incertidumbre.

Factor de control interno, entendido en los negocios, como una forma de influir en los resultados empresariales. Este factor se evaluó con las siguientes opciones:

- Normalmente realizo muy bien mi parte de trabajo en cualquier proyecto en el que estoy implicado.

- Si quiero algo, trabajo duro para conseguirlo.

Factor de autoestima, mide la confianza y sentimiento de competencia para llevar a cabo el negocio. Se evaluó con los siguientes enunciados:

- Estar auto empleado implica mayores riesgos que los que estoy dispuesto a correr.

- Creo que lo más importante a la hora de seleccionar socios es que sean competentes.

- Me siento satisfecho cuando hago algo que permite que el grupo o las organizaciones a las que pertenezco funcionen mejor.

Factores de apoyo, adicional a los factores explicados, se consultó a los estudiantes acerca de los elementos de apoyo que esperan provengan de la academia, para ello se plantearon las siguientes opciones en la encuesta:

- El impedimento más importante para que se pueda poner en marcha un negocio propio es.

- El apoyo más importante que necesita un estudiante o graduado de la universidad para que un negocio propio sea exitoso es.

- El tema más importante que debería ofertarse en Inter ciclos para que un proyecto se convierta en una empresa exitosa es.

\section{Resultados}

Los resultados de las encuestas aplicadas a los estudiantes de la Facultad de Hospitalidad y Servicios se exponen de acuerdo al factor investigado, así:

En el factor necesidad de logro se observa la dedicación de tiempo para desarrollar 
nuevas oportunidades (63\% total acuerdo), el trabajo duro (80\% total acuerdo) y $40 \%$ en total desacuerdo en que el tiempo libre es más importante que los retos y las nuevas oportunidades, indican que la necesidad de logro es una importante tendencia en los estudiantes. (Ver tabla 4).

\section{Tabla 4}

Resultados Factor Necesidad de Logro

\begin{tabular}{|c|c|c|c|c|c|}
\hline Enunciado & \begin{tabular}{l}
\multicolumn{1}{c}{ Creo que } \\
la gente con \\
éxito se \\
manejará \\
mejor que \\
yo en \\
reuniones \\
de \\
negocios.
\end{tabular} & $\begin{array}{l}\text { Las horas } \\
\text { regulares de } \\
\text { trabajo y las } \\
\text { vacaciones son } \\
\text { más importantes } \\
\text { para mí que las } \\
\text { nuevas } \\
\text { oportunidades y } \\
\text { retos que ofrezca } \\
\text { un puesto. }\end{array}$ & $\begin{array}{l}\text { Creo que para } \\
\text { tener éxito en un } \\
\text { negocio debes } \\
\text { dedicar tiempo } \\
\text { todos los días a } \\
\text { desarrollar nuevas } \\
\text { oportunidades. }\end{array}$ & $\begin{array}{l}\text { Creo que es } \\
\text { necesario } \\
\text { pasar mucho } \\
\text { tiempo } \\
\text { planeando las } \\
\text { actividades } \\
\text { empresariales }\end{array}$ & $\begin{array}{l}\text { Me siento } \\
\text { bien cuando } \\
\text { he trabajado } \\
\text { duro para } \\
\text { mejorar mi } \\
\text { trabajo }\end{array}$ \\
\hline $\begin{array}{c}\text { Total } \\
\text { desacuerdo }\end{array}$ & $21.2 \%$ & $39.9 \%$ & $1.3 \%$ & $0.9 \%$ & $0.3 \%$ \\
\hline $\begin{array}{l}\text { Bastante en } \\
\text { desacuerdo }\end{array}$ & $17.4 \%$ & $20.6 \%$ & $1.9 \%$ & $2.5 \%$ & $1.6 \%$ \\
\hline $\begin{array}{l}\text { Parcialmente } \\
\text { en desacuerdo }\end{array}$ & $21.2 \%$ & $20.6 \%$ & $0.3 \%$ & $7.6 \%$ & $0.6 \%$ \\
\hline $\begin{array}{l}\text { Parcialmente } \\
\text { de acuerdo }\end{array}$ & $23.4 \%$ & $8.2 \%$ & $7.0 \%$ & $38.6 \%$ & $3.2 \%$ \\
\hline $\begin{array}{l}\text { Bastante de } \\
\text { acuerdo }\end{array}$ & $10.4 \%$ & $5.1 \%$ & $26.6 \%$ & $34.8 \%$ & $14.6 \%$ \\
\hline Total acuerdo & $6.3 \%$ & $5.7 \%$ & $63.0 \%$ & $15.5 \%$ & $79.7 \%$ \\
\hline
\end{tabular}

Fuente: Elaboración propia

En el factor de innovación se observa una buena actitud a asumir riesgos y predisposición a la innovación, los mayores porcentajes de aceptación de la escala de Likert se ve en los aspectos favorables que sobresalen como: la mucha necesidad de nuevas aventuras, $51 \%$ total acuerdo, la motivación que les mueve al pensar en nuevas ideas que estimulen un negocio, $48 \%$ total acuerdo, y el apostar por nuevas ideas, aunque no sean del todo seguras, $39 \%$ parcialmente de acuerdo. (Ver tabla 5). 


\section{Tabla 5}

Resultados Factor de Innovación

\begin{tabular}{|c|c|c|c|c|c|c|c|c|}
\hline Enunciado & $\begin{array}{l}\text { Me gusta } \\
\text { que las } \\
\text { cosas } \\
\text { sean } \\
\text { estables } \\
\text { y } \\
\text { predecibl } \\
\text { es }\end{array}$ & $\begin{array}{l}\text { Me gusta } \\
\text { trabajar } \\
\text { dónde no } \\
\text { hay mucha } \\
\text { incertidum } \\
\text { bre ni } \\
\text { cambios } \\
\text { constantes }\end{array}$ & \begin{tabular}{l}
\multicolumn{1}{c}{ No me } \\
molesta la \\
incertidum \\
bre y el \\
riesgo que \\
suele \\
provocar lo \\
desconocid \\
o.
\end{tabular} & $\begin{array}{l}\text { Suelo } \\
\text { apostar } \\
\text { por } \\
\text { buenas } \\
\text { ideas, } \\
\text { aunque } \\
\text { no sean } \\
\text { del todo } \\
\text { seguras }\end{array}$ & \begin{tabular}{l}
\multicolumn{1}{c}{ Tengo } \\
mucha \\
necesida \\
d de \\
nuevas \\
aventuras \\
.
\end{tabular} & \begin{tabular}{l}
\multicolumn{1}{c}{ Lo que } \\
verdadera \\
mente me \\
motiva es \\
pensar en \\
nuevas \\
ideas que \\
estimulen \\
un \\
negocio
\end{tabular} & $\begin{array}{l}\text { Por lo } \\
\text { general } \\
\text { evito } \\
\text { asumir } \\
\text { riesgos. }\end{array}$ & \begin{tabular}{l}
\multicolumn{1}{c}{ Vivo } \\
cómodo \\
en \\
situacion \\
es de \\
cambio e \\
incertidu \\
mbre.
\end{tabular} \\
\hline $\begin{array}{c}\text { Total } \\
\text { desacuerdo }\end{array}$ & $4 \%$ & $11 \%$ & $4 \%$ & $2 \%$ & $1 \%$ & $0 \%$ & $17 \%$ & $9 \%$ \\
\hline $\begin{array}{l}\text { Bastante } \\
\text { en } \\
\text { desacuerdo }\end{array}$ & $6 \%$ & $6 \%$ & $6 \%$ & $4 \%$ & $1 \%$ & $2 \%$ & $26 \%$ & $13 \%$ \\
\hline $\begin{array}{l}\text { Parcialme } \\
\text { nte en } \\
\text { desacuerdo }\end{array}$ & $12 \%$ & $11 \%$ & $10 \%$ & $9 \%$ & $2 \%$ & $1 \%$ & $23 \%$ & $24 \%$ \\
\hline $\begin{array}{l}\text { Parcialme } \\
\text { nte de } \\
\text { acuerdo }\end{array}$ & $32 \%$ & $30 \%$ & $27 \%$ & $39 \%$ & $19 \%$ & $12 \%$ & $19 \%$ & $30 \%$ \\
\hline $\begin{array}{c}\text { Bastante } \\
\text { de acuerdo }\end{array}$ & $24 \%$ & $22 \%$ & $26 \%$ & $30 \%$ & $27 \%$ & $38 \%$ & $8 \%$ & $16 \%$ \\
\hline $\begin{array}{c}\text { Total } \\
\text { acuerdo }\end{array}$ & $22 \%$ & $19 \%$ & $26 \%$ & $16 \%$ & $51 \%$ & $47 \%$ & $7 \%$ & $9 \%$ \\
\hline
\end{tabular}

Fuente: Elaboración propia

En el factor control interno también se observa una buena predisposición para el trabajo arduo que un nuevo emprendimiento exige, así lo demuestran las respuestas cuando trabajan duro para conseguir algo, $77 \%$ total acuerdo y la creencia de que una organización puede llegar a ser más efectiva con personal competente, $52 \%$ total acuerdo. (Ver tabla 6).

Tabla 6

Resultados Factor Control Interno

\begin{tabular}{|c|c|c|}
\hline Enunciado & $\begin{array}{l}\text { Normalmente realizo muy } \\
\text { bien mi parte de trabajo en } \\
\text { cualquier proyecto en el que } \\
\text { estoy implicado. }\end{array}$ & $\begin{array}{l}\mathrm{Si} \text { quiero algo, trabajo } \\
\text { duro para conseguirlo. }\end{array}$ \\
\hline \multicolumn{3}{|l|}{ Variable } \\
\hline Total desacuerdo & $1.9 \%$ & $2.22 \%$ \\
\hline $\begin{array}{l}\text { Bastante en } \\
\text { desacuerdo }\end{array}$ & $0.63 \%$ & $1.58 \%$ \\
\hline $\begin{array}{l}\text { Parcialmente en } \\
\text { desacuerdo }\end{array}$ & $0.63 \%$ & $4.11 \%$ \\
\hline $\begin{array}{l}\text { Parcialmente de } \\
\text { acuerdo }\end{array}$ & $4.11 \%$ & $12.97 \%$ \\
\hline Bastante de acuerdo & $15.51 \%$ & $27.22 \%$ \\
\hline Total acuerdo & $77.22 \%$ & $51.90 \%$ \\
\hline
\end{tabular}

Fuente: Elaboración propia 
En el factor autoestima se observa cierta debilidad, en especial al evaluar la percepción de ser auto empleado, $22 \%$ parcialmente de acuerdo, sin embargo, en el nivel de satisfacción cuando contribuyen a que el grupo u organización a la que pertenecen funcionen mejor se observa un resultado de $69 \%$ en total acuerdo. (Ver tabla 7).

\section{Tabla 7}

Resultados Factor Autoestima

\begin{tabular}{|c|c|c|c|}
\hline Enunciado & $\begin{array}{l}\text { Estar auto } \\
\text { empleado implica } \\
\text { mayores riesgos que } \\
\text { los que estoy } \\
\text { dispuesto a correr. }\end{array}$ & $\begin{array}{l}\text { Creo que lo más } \\
\text { importante a la hora } \\
\text { de seleccionar } \\
\text { socios es que sean } \\
\text { competentes. }\end{array}$ & $\begin{array}{l}\text { Me siento satisfecho } \\
\text { cuando hago algo que } \\
\text { permite que el grupo o } \\
\text { las organizaciones a } \\
\text { las que pertenezco } \\
\text { funcionen mejor. }\end{array}$ \\
\hline Total desacuerdo & $16.1 \%$ & $0.3 \%$ & $0.6 \%$ \\
\hline $\begin{array}{l}\text { Bastante en } \\
\text { desacuerdo }\end{array}$ & $19 \%$ & $1.9 \%$ & $0.6 \%$ \\
\hline $\begin{array}{l}\text { Parcialmente en } \\
\text { desacuerdo }\end{array}$ & $20.3 \%$ & $4.4 \%$ & $1.3 \%$ \\
\hline $\begin{array}{l}\text { Parcialmente de } \\
\text { acuerdo }\end{array}$ & $21.8 \%$ & $17.1 \%$ & $5.1 \%$ \\
\hline Bastante de acuerdo & $10.8 \%$ & $33.9 \%$ & $23.7 \%$ \\
\hline Total acuerdo & $12 \%$ & $42.4 \%$ & $68.7 \%$ \\
\hline
\end{tabular}

Fuente: Elaboración propia

Dentro de los factores de apoyo, en cuanto a los impedimentos más importantes para poner en marcha un negocio propio, los estudiantes manifestaron la capacidad económica con un $43 \%$ y el miedo al fracaso con un $31 \%$. (Ver tabla 8).

\section{Tabla 8}

Resultados Impedimentos para un negocio propio

\begin{tabular}{lc}
\hline Enunciado & $\begin{array}{c}\text { El impedimento más importante } \\
\text { para que se pueda poner en marcha } \\
\text { un negocio propio es }\end{array}$ \\
Variable & $43 \%$ \\
\hline Capacidad económica & $8 \%$ \\
Falta de apoyo & $17 \%$ \\
Falta de conocimiento & $31 \%$ \\
Miedo al fracaso & $1 \%$ \\
No me interesa tener un negocio & \\
propio & \\
\hline
\end{tabular}

Fuente: Elaboración propia

En la interrogante sobre cuál es el apoyo más importante que necesita un estudiante o graduado de la universidad para que un negocio propio sea exitoso, un 38,3\% dijo que necesitan capital de pre incubación, o sea, inyección de dinero para estudios de mercado, desarrollo de prototipos, innovación en los productos/servicios y un 33,5\% solicitan capital semilla, es decir, inyección de dinero para el inicio del negocio. (Ver tabla 9). 


\section{Tabla 9}

Resultados Apoyo más importante de la Universidad

\begin{tabular}{lc}
\hline Enunciado & $\begin{array}{c}\text { El apoyo más importante que } \\
\text { necesita un estudiante o graduado de } \\
\text { la universidad para que un negocio } \\
\text { propio sea exitoso es: }\end{array}$ \\
Variable & $8.5 \%$ \\
\hline Capacitación especializada \\
(materias optativas impartidas en \\
$\begin{array}{l}\text { interciclos) } \\
\text { Capital de pre incubación }\end{array}$ \\
(inyección de dinero para estudios \\
de mercado, desarrollo de \\
prototipos, innovación en los \\
productos/servicios) \\
$\quad \begin{array}{l}\text { Capital de riesgo (inyección de } \\
\text { dinero para el crecimiento del }\end{array}$ \\
negocio) \\
$\quad$ Capital semilla (inyección de \\
dinero para el inicio del negocio) \\
$\quad$ Contacto con empresarios
\end{tabular}

Fuente: Elaboración propia

Respecto a la formación que requieren, un 32,3\% manifestó en planes de negocios y un $26,3 \%$ en desarrollo de prototipos con componentes innovadores/diferenciadores. (Ver tabla 10).

\section{Tabla 10}

Resultados Tema más importante para impartirse en interciclos

\begin{tabular}{ll}
\hline Enunciado & $\begin{array}{c}\text { El tema más importante que } \\
\text { debería ofertarse en interciclos para } \\
\text { que un proyecto se convierta en una } \\
\text { empresa exitosa es: }\end{array}$ \\
Variable & $20.9 \%$ \\
\hline$\quad$ Cultura emprendedora & $26.3 \%$ \\
$\quad \begin{array}{l}\text { Desarrollo de prototipos con } \\
\text { componentes } \\
\text { innovadores/diferenciadores } \\
\quad \text { Elaboración de plan de negocios }\end{array}$ & $32.3 \%$ \\
$\quad$ Localización de fuentes de & $11.1 \%$ \\
financiamiento & $9.5 \%$ \\
$\quad$ Técnicas de ventas &
\end{tabular}

Fuente: Elaboración propia

\section{Discusión}

Ante la carencia de estrategias de apoyo provenientes de la academia, los estudiantes pueden desechar sus ideas o buscar en otros espacios alguna organización que crea en sus ideas de negocio. Se desconoce así cuantas ideas de negocio se han desperdiciado y también es difícil cuantificar el número de empresas que han sobrevivido a las inclemencias del mercado y de otras incertidumbres que atentan la permanencia de negocios creados con iniciativas estudiantiles. 
Las universidades hacen esfuerzos aislados para que esta situación cambie, pero realmente se requiere la participación de algunos actores que interactúen en favor de una cultura emprendedora. Así mismo, el gobierno intenta a través de sus programas que exista un impacto positivo en la transferencia del conocimiento y tecnología, procura concertar entre la empresa privada y la academia, reconociéndose insuficientes logros en comparación a otros países en donde este sistema Universidad - Empresa privada y Gobierno funciona en forma consolidada y competitiva.

En la región se reconoce que el emprendimiento crea desarrollo económico importante, crea empleo y mejora la calidad de vida de la población, sin embargo, al menos en nuestro medio, la academia no es un agente que implemente estrategias efectivas para ello, esta eficiencia debe nacer a partir de una reforma en la normativa estatutaria al interior de las universidades y a partir de esta nueva normativa se incorporen en los currículos temas que afiancen la cultura emprendedora, promuevan el desarrollo de prototipos, permitan incorporar mecanismos de financiamiento y de recuperación de la inversión que apuesten en las ideas estudiantiles.

\section{Conclusiones}

Los estudiantes de esta facultad tienen una alta predisposición para trabajar duro, en forma independiente y con un esfuerzo extraordinario, esta actitud puede ser demostrada medianamente al percibirse como autoempleados y en especial siendo empleados.

Medianamente tienen inclinación a la innovación, probablemente por desconocer conceptos, habilidades y el camino técnico que debe seguir la innovación, situación que se debería potenciar introduciendo en la facultad la cultura del emprendimiento y también una cultura de innovación, siempre orientados con técnicos conocedores en la materia.

Definitivamente el tema de autoestima hay que fortalecer en los estudiantes, para crear seguridad, liderazgo e impregnar de habilidades características de los emprendedores triunfadores. No todos tienen la actitud, hay que enfocar los esfuerzos a quienes demuestren persistencia y asunción al riesgo.

Incipientes iniciativas del Estado están a la vista, lo que falta es presencia por parte de la industria y un mecanismo adecuado que integre a estos actores. Este mecanismo es un ecosistema de negocios en donde el ambiente sea el propicio para que Universidad, Estudiantes, Empresa y Gobierno interactúen en forma coordinada y eficiente.

Una debilidad del estudiante es que tienen inseguridad de administrar exitosamente su empresa, sin embargo, tienen buena predisposición a la aventura e innovación lo que indica la existencia de un importante potencial en los estudiantes de esta facultad para la generación de proyectos dinámicos.

Como factores de apoyo que deben provenir de la academia se establecen los siguientes: generación de una cultura emprendedora, transferencia de conocimientos a la industria, direccionar a los emprendedores según los roles dentro de un ecosistema de negocios, conocer 
las instituciones relacionadas para el apoyo a emprendedores, existencia de un fondo para emprendimiento y capacitación en financiamiento.

Otro factor a considerar es la necesidad de incorporar componentes tecnológicos y de ingeniería en los diseños de prototipos que posteriormente se convertirán en productos y servicios con características innovadoras con alto aprecio en el mercado.

Como estrategias destacables que deba considerar la Universidad UTE para el fomento sostenible del emprendimiento estudiantil son: Integración activa a redes de emprendimiento e innovación dentro de un contexto nacional y regional; incluir en el plan estratégico institucional objetivos de emprendimiento estudiantil; conformar un comité emprendedor multidiscipliario con representantes de varios estamentos para que propongan una hoja de ruta y un sistema de seguimiento por resultados, así también puede encargarse de crear una cultura de emprendimiento como política institucional, búsqueda de capital semilla y capital de riesgo.

Al interior de la Facultad de Hospitalidad y Servicios iniciar con la formación de formadores en emprendimiento, dotar los recursos necesarios para ofrecer capacitaciones en las jornadas de interciclos, como materias optativas, en temas especializados como plan de negocios, patentes, mercadeo y conexión con inversionistas que financien los prototipos de media y alta tecnología con la participación de técnicos que manejen los laboratorios institucionales a favor de los proyectos innovadores.

\section{Implicaciones futuras}

Este estudio trae las siguientes implicaciones para investigaciones futuras:

- Redes de emprendimientos en tecnología media y tecnología avanzada.

- Empresas Spin off como un medio de desarrollo de emprendimiento estudiantil y participación empresarial para la Universidad, bienes sustitutos de importaciones sería el enfoque para la innovación.

\section{Bibliografía}

Attitude, E., Students, O. F., The, O. F., \& Year, L. (2018). Enterprising Attitude Of Students Of The Last Year Of Acade-, 45, 69-79.

Canto, M. S., \& Herrera, V. (2018). Implementación del Emprendimiento en la Universidad Tecnológica de Panamá, y el Perfil del Emprendedor Universitario. KnE Engineering, 3(1), 246. https://doi.org/10.18502/keg.v3i1.1430

De la Fuente Arias, J., Vera Martínez, M. M., \& Cardelle-Elawar, M. (2017). Aportaciones de la Psicología de la Innovación y del Emprendimiento a la Educación, en la Sociedad del Conocimiento. Electronic Journal of Research in Education Psychology, 10(28). https://doi.org/10.25115/ejrep.v10i28.1544

Echeverri-Sanchez, L., Valencia-Arias, A., Benjumea-Arias, M., \& Barrera-Del Toro, A. (2018). Factores que inciden en la intención emprendedora del estudiantado universitario: Un análisis cualitativo. Revista Electrónica Educare, 22(2), 1. https://doi.org/10.15359/ree.222.10 
Emprendedora, S. C. (2006). Forjando emprendedores.

Espíritu Olmos, R., \& Sastre Castillo, M. Á. (2007). La actitud emprendedora durante la vida académica de los estudiantes universitarios. Cuadernos de Estudios Empresariales, 17(January), 95-116.

García-García, V. D. (2015). Youth Entrepreneurship: An assessment with university students. Revista Latinoamericana de Ciencias Sociales, Niñez y Juventud, 13(2), 1221-1236. https://doi.org/10.11600/1692715x.13246200315

Guerrero Maribel, \& Urbano David. (1992). Knowledge and Technology Transfer Strategies: Best Practices in Spanish Entrepreneurial Universities. Gestión y Política Pública, 21(1), 107139. Retrieved from http://www.scielo.org.mx/scielo.php?script=sci_arttext\&pid=S140510792012000100004

Hernando, J., \& Mitchell, D. (2017). Innovación y emprendimiento en Colombia: balance, perspectivas y recomendaciones de política, 2014-2018. Cuadrernos Fedesarrollo, 94. Retrieved from file:///C:/Users/Andy/Documents/importaciones 1.pdf

Hidalgo, L. F. (2015). La cultura del emprendimiento y su formación The Culture of Entrepreneurship and Education, 3(2).

Jacóme, I., Tinajero, M., \& Suárez, I. (2018). Características del comportamiento emprendedor en estudiantes egresados universitarios del Ecuador. Dominio de Las Ciencias, ISSN-e 24778818, Vol. 4, $N^{o}$. 3, 2018, Págs. 163-176, 4(3), 163-176. Retrieved from https://dialnet.unirioja.es/servlet/articulo?codigo $=6560193$

Liévano, J., Vargas, P., Pico, B., \& Pérez, J. (2018). Importancia De La Innovación Para Emprendedores De Micro Y Pequeñas Empresas Mexicanas Del Sector Servicio En Villahermosa-Tabasco Y Mexicali-Baja California. Estudio Cuantitativo. European Scientific Journal, 14(22), 304-320. https://doi.org/10.19044/esj.2018.v14n22p304

Marquinez, T. (2018). Facultad De Ciencias Económicas Y Gestión Empresarial Internacional Tema: Actitudes emprendedoras de los estudiantes universitarios: Caso Universidad Católica de Santiago de Guayaquil AUTORA : Marquinez Mite Tania Gabriela Trabajo de titulación previo a. Retrieved from http://repositorio.ucsg.edu.ec/handle/3317/9919

Montoya, M., \& Casas, J. (2015). Características para promover el emprendimiento y la innovación. Marco general para la formación en las universidades. UPIICSA. Investigación Interdisciplinaria, 1(2), 32-45. Retrieved from http://www.ruii.ipn.mx

Orozco, L., \& Chavarro, D. (2008). Universidad Y Emprendimiento. Hallazgos, (10), 65-97. Retrieved from http://www.redalyc.org/articulo.oa?id=413835171006

Perez, E. (2009). La universidad en la formación de emprendedores empresariales y el apoyo en la creación de nuevas empresas. Revista de Investigación de La Facultad de Ciencias Administrativas, 12(23), 61-65. Retrieved from http://revistasinvestigacion.unmsm.edu.pe/index.php/administrativas/article/view/8912/7 740

Revelo, R., Lucio, B., Mata, A., \& Romero, E. (2017). Medición de la actitud emprendedora en los estudiantes de la Universidad Central del Ecuador. Revista Publicando, (111), 422434.

Robinson, P. B., Stimpson, D. V., Huefner, J. C., \& Hunt, H. K. (2018). An Attitude Approach to the Prediction of Entrepreneurship. Entrepreneurship Theory and Practice, 15(4), 13-32. https://doi.org/10.1177/104225879101500405

Saldaña, D. E., \& Coutiño, R. S. (2010). Universidad y emprendimiento social: El caso del Tecnológico de Monterrey. ICADE. Revista Cuatrimestral de Las Facultades de Derecho 
y Ciencias Económicas y Empresariales, (80), 265-290.

Sanabria Rangel, P. E., Morales Rubiano, M. E., \& Ortiz Riaga, C. (2015). Interacción Universidad y entorno: marco para el emprendimiento. Educación y Educadores, 18(1), 111-134. https://doi.org/10.5294/edu.2015.18.1.7

Souza, R. D. S., \& Silveira, A. (2018). Intención emprendedora en contexto universitario brasileño. Revista Gestão Universitária Na América Latina - GUAL, 11(2), 297-318. https://doi.org/10.5007/1983-4535.2018v11n2p297

Tarapuez, E., García, M. D., \& Castellano, N. (2018). Aspectos socioeconómicos e intención emprendedora en estudiantes universitarios del Quindío (Colombia). Innovar, 28(67), 123135. https://doi.org/10.15446/innovar.v28n67.68618 